\title{
Perception of simultaneous simulations of two rotating spheres in the same visual locale
}

\author{
J. TIMOTHY PETERSIK \\ Southeast Missouri State University, Cape Girardeau, Missouri 63701
}

\begin{abstract}
In many three-dimensional (3-D) simulations of a rotating object, the perceptual task facing the observer is formidable. Nonetheless, the spontaneous percept is usually coherent; that is, a rigid 3-D object rotating in depth is perceived. In the present experiment, observers viewed a stroboscopic display consisting of 100 randomly located luminous dots that simulated the simultaneous rotation of two concentric spheres. Under appropriate conditions of presentation, observers could spontaneously discriminate the relative rotations of the two spheres. Phenomenal reports of the subjects were also recorded.
\end{abstract}

Petersik (1979) has reported two experiments that demonstrate that the three-dimensional (3-D) percepts that result from computer-animated dynamic perspective simulations of rotating spheres are remarkably insensitive to the addition of visual noise. In one experiment, the internal volume of a sphere was specified by the apparent movement and perspective transformations of short vectors. A change from frame to frame in the orientation of the vectors that composed the simulation reduced, but did not destroy, the perceived depth of the sphere and the subjects' rotation judgment accuracy. In the remaining experiment, the display consisted of 50 small luminous dots. A certain number of these dots were continuously transformed and moved according to the rules of perspective (the signal dots). The signal dots therefore specified the structure and rotation of a transparent sphere partially filled with luminous dots. However, the remaining dots of the simulation simply moved to and fro randomly across the same area of the display as that covered by the projection of the sphere. These randomly moving dots constituted two-dimensional (2-D) noise. Displays consisting of as little as 9\% signal dots and $91 \%$ noise dots were found to elicit accurate rotation-direction judgments, as well as quantitatively and qualitatively "good" sensations of the volume of the simulated sphere. Such displays were so surprising and compelling that we tried to find a source of "structured noise" that might interfere with the visual system's ability to detect dynamic perspective transformations and to consequently construct 3-D percepts of objects in rotation. Accordingly, we created new computeranimated displays in which the simultaneous rotation of two concentric spheres was simulated.

The present study was conducted in the laboratory of Allan Pantle at Miami University. The author is indebted to Dr. Pantle for his donation of space and equipment. Many thanks are due Kimberly Kurtz for her patient service as an observer. Requests for reprints should be addressed to J. Timothy Petersik, Department of Psychology, Southeast Missouri State University, Cape Girardeau, Missouri 63701.
In order to understand the rationale for these displays more clearly, the reader is asked to imagine the projection of two stationary transparent spheres, each of which contains 50 luminous dots within its volume. Both spheres occupy the same region of the visual field. With stationary spheres and a stationary observer, the percept will be of a flat circular surface randomly dotted with luminous points. Next, imagine the polar projections produced when both spheres begin to rotate. In the projections, approximately half of all dots will be moving from left to right at any point in time. However, half of those left-to-right dots will "belong" to one sphere, while half will belong to the other. Similarly, half of all right-to-left moving dots will belong to each sphere. The task of perceptually constructing two coherent spheres from such information is compounded by the fact that the two spheres may be rotating in either the same or opposite directions. If both concentric spheres rotate in the same direction and at the same angular speed, then, in the projections of the spheres, all of the dots moving in a single direction (e.g., right to left) will belong in the same half of their respective spheres; that is, they will all belong in either the "front" or "rear" half of the respective spheres, depending upon whether the rotation is clockwise or counterclockwise. On the other hand, if the spheres are rotating in opposite directions, in the projections of the spheres, some of the dots moving in a given direction will belong in the front half of one of the spheres, while other dots moving in the same direction will belong in the rear half of the other sphere. With such seemingly incompatible perspective information distributed over the same area of the visual field, one might expect that an observer could not easily and spontaneously segregate the moving dots into two simultaneously rotating spheres. However, data from the present experiment show that when simulations of two simultaneously rotating spheres are viewed, the resulting percept is frequently of two distinct spheres, each with its own unique 3-D structure and rotation direction. Further- 
more, regardless of their subjective impressions of such displays, observers can almost always report whether the two spheres rotate in the same or opposite directions.

\section{METHOD}

\section{Stimuli and Apparatus}

Stimuli for the present experiment were computer-animated simulations of the polar projections of the simultaneous rotations of two transparent spheres partially filled with randomly positioned luminous dots. ${ }^{1}$ A computer-animation method described by Braunstein (1976) was adapted to produce these simulations. In general, the computer first creates the internal representations of the 3-D locations of several points within two distinct concentric spheres and then determines their 2-D projections. The 2-D projections are then stored as one "frame" of a simulation. After this has been done a number of times for the same two spheres in each of a number of equally spaced positions of a cycle of rotation, the computer has constructed a "movie" of the rotating spheres. Such display sequences were created on-line during the course of the experiment by a PDP-11/10 minicomputer (DEC), and they were displayed by the same computer on the face of an oscilloscope coated with P31 phosphor. Each of the simulated spheres contained 50 dots, so that the entire display consisted of 100 moving dots. A new random arrangement of the positions of the dots was established for each new simulation, so that no recognizable subpatterns of dots existed over trials. When viewed from a distance of $200 \mathrm{~cm}$, each constituent dot subtended a visual angle of $1.5 \mathrm{~min}$. The luminance of each component dot was $4.39 \mathrm{~cd} / \mathrm{m}^{2}$.

Each simulation consisted of only 30 frames, so that the positions of the spheres in their cycles of rotation changed by $12 \mathrm{deg}$ from frame to frame. The frame duration of each simulation was $100 \mathrm{msec}$, whereas the interframe interval (IFI) varied between 10 and $100 \mathrm{msec}$.

One of the spheres in each simulation (the $C$ sphere) always remained constant with respect to two parameters of presentation, namely, size and simulated projection distance. The diameter of the $\mathrm{C}$ sphere subtended $2 \mathrm{deg}$ visual angle. The $\mathrm{C}$ sphere was constructed to simulate the perspective information of a physical sphere viewed from a distance of 3.0 sphere radii from the center of the sphere. That is, the simulated projection distance was 3.0 sphere radii. The second sphere of any simulation (the $\mathrm{V}$ sphere) was variable with respect to the size and simulated projection-distance parameters. For one-third of all trials, the diameter of the V sphere subtended 1,2 , or $2.9 \mathrm{deg}$ visual angle. On any trial, the simulated projection distance of the $\mathrm{V}$ sphere was $1.0,1.5,2.0,2.5,3.0,3.5$, or 4.0 sphere radii. Viewing distance was not varied to accommodate changes in simulated projection distanœ, since Hershberger and Urban (1970), using a similar type of rotation simulation, have determined that "the veridicality of judgments of direction of rotation varied as an inverse function of [simulated] projection distance and was unaffected by viewing distance" (p. 135) (see also Petersik, 1978).

Since the size and dot density of the V sphere varied among three levels, the space-average luminance of these displays also varied from condition to condition. However, pilot research (Petersik, 1978) has shown that luminance variations between threshold and over 1 log unit above threshold do not affect the quality of the percepts, nor the performance of subjects on objective measures. In the present experiment, the luminance of the displays was always above threshold and never reached a level of 1 log unit above threshold.

Details regarding these displays are presented elsewhere (Petersik, 1978, 1979). In the present experiment, each simulation was invisibly constructed by the computer prior to each trial and was stored in memory in the form of subpictures; the entire sequence was displayed upon completion of the final subpicture. Each display sequence was preceded by a 3-sec blinking warning light.

\section{Subjects}

Subjects were two highly practiced psychophysical observers, each of whom had a minimum of $30 \mathrm{~h}$ experience viewing single-sphere rotation simulations prior to this experiment. Observer K.K. was an undergraduate volunteer who had no knowledge of the purpose of the experiment. Observer T.P. was the author.

\section{Procedure}

Each subject served in 10 experimental sessions, each held on a different day. The computer controlled each experimental session and recorded responses. Displays were viewed with both eyes and without a fixation point, except for the warning light that appeared at the screen location corresponding to the center of the simulated rotating spheres. Each subject sat directly in front of the display oscilloscope at a distance of $200 \mathrm{~cm}$ and minimized head and eye movements. The oscilloscope was approximately at eye level. Each display was viewed in a nearly dark room. There was some ambient illumination between trials when the subject used a night-light to view the computer Teletype keyboard. Each subject viewed the simulations globally without fixating local areas of the display.

During a single experimental session, a subject viewed one block of 42 different displays resulting from the factorial combination of three V-sphere sizes, seven simulated V-sphere sizes, seven simulated projection distances, and two interframe intervals (IFIs). The order of presentation was random. On each trial the computer randomly determined the rotation direction of each sphere, yielding an a priori probability of .5 that the two spheres would rotate in the same direction. Immediately following each trial, the subject pressed one of two keys to indicate whether the two spheres rotated in the same or opposite directions. Also, the phenomenal observations of each subject were recorded. About $30 \mathrm{sec}$ elapsed between trials as the computer constructed the next sequence of simulation frames. No feedback was provided after trials.

The objective data were analyzed by analysis of variance procedures.

\section{RESULTS}

\section{Phenomenal Observations}

Despite the complexity of the information contained in the present displays, in the 10-msec IFI conditions, subjects had little difficulty establishing and maintaining a constant and coherent percept of the 3-D structure of at least one of the two spheres. On at least half of the 10-msec IFI trials, two 3-D spheres were perceived in simultaneous rotation, regardless of whether their rotations were in the same or opposite directions. On the remaining trials, the 3-D structure of the second sphere "faded in and out." On a few trials, the structure of the second sphere was never perceived.

Generally, none of the spheres in the $100-\mathrm{msec}$ IFI conditions appeared as complete or full as the spheres in the $10-\mathrm{msec}$ IFI conditions. In the $100-\mathrm{msec}$ IFI conditions, the 3-D structure of both spheres was rarely perceived at one time. On approximately onethird of all 100-msec IFI trials, no 3-D sphere was perceived. However, the observers had occasional sensations of structureless depth on such trials. On onethird of the remaining trials, at least one sphere rotating in depth was perceived, although, as mentioned, it was not as "good" (in the Gestalt sense) as spheres in the $10-\mathrm{msec}$ IFI conditions. Finally, on the remaining one- 
third of the 100-msec IFI trials, two spheres were perceived, but they only rarely appeared simultaneously. It was occasionally noted that such spheres fragmented and temporarily lost all structure. Across all conditions, perception of the $\mathrm{V}$ sphere seemed to be easiest when its diameter was largest.

\section{Same-Different Judgments}

Table 1 shows the results of this experiment with respect to the IFI and V-sphere size variables, collapsed over simulated projection distance, for each subject. Each entry shows the percentage of correct samedifferent judgments for a particular combination of IFI and V-sphere size. As might be expected given the phenomenal reports, the $10-\mathrm{msec}$ IFI conditions resulted in better relative rotation discrimination than did the 100-msec IFI conditions. When collapsed across all other variables, the average difference in the percentage of correct same-different judgments between the 10-msec and $100-\mathrm{msec}$ IFI conditions was $13.7 \%$. However, in spite of the poor subjective percepts produced by the simulations in the 100-msec IFI conditions, and in spite of the inferior discrimination performance that they elicited, four of the six $100-\mathrm{msec}$ entries in Table 1 are nonetheless 3 or more standard errors above a chance level of responding. This finding suggests, and it was also the subjective impression of the subjects, that rotation can be detected in the absence of the perception of 3-D structure. Table 1 also shows that, at least with respect to the $10-\mathrm{msec}$ IFI simulations, relative rotation discrimination improved as a monotonic function of $\mathrm{V}$-sphere size. These findings are somewhat counterintuitive, since one might expect that when the $\mathrm{V}$ sphere and $\mathrm{C}$ sphere are identical in size they would be the most difficult to segregate perceptually.

Figure 1 shows, for each of the three sizes of the $\mathrm{V}$ sphere, the mean percentage of correct same-different judgments as a function of the simulated projection distance in the 10-msec IFI displays. Since the 100-msec IFI displays did not elicit phenomenally good percepts of two rotating spheres, the objective data from those conditions were not analyzed further.

The first observation of interest regarding Figure 1 is that no combination of V-sphere size and simulated projection distance elicited chance performance from the two subjects. Especially for the largest V-sphere size ( $2.9 \mathrm{deg}$, solid squares), the relative rotation of the two simulated spheres was nearly always detected. As shown

Table 1

Mean Percentage of Correct Same-Different Judgments as a Function of Sphere Size (in Degrees) and IFI

\begin{tabular}{lcccccccc}
\hline & \multicolumn{3}{c}{10 -msec IFI } & & \multicolumn{3}{c}{100 -msec IFI } \\
\cline { 2 - 4 } \cline { 6 - 8 } Subject & 1 & 2 & 2.9 & & \multicolumn{3}{c}{ Sphere Size } \\
K.K. & 85.7 & 86.1 & 90.0 & & 68.6 & $67.1^{*}$ & 72.9 \\
T.P. & 74.3 & 77.1 & 92.9 & & $61.4^{*}$ & 72.9 & 81.4 \\
\hline
\end{tabular}

*Percentages less than 3 standard errors above chance (all others are at least 3 standard errors above chance).

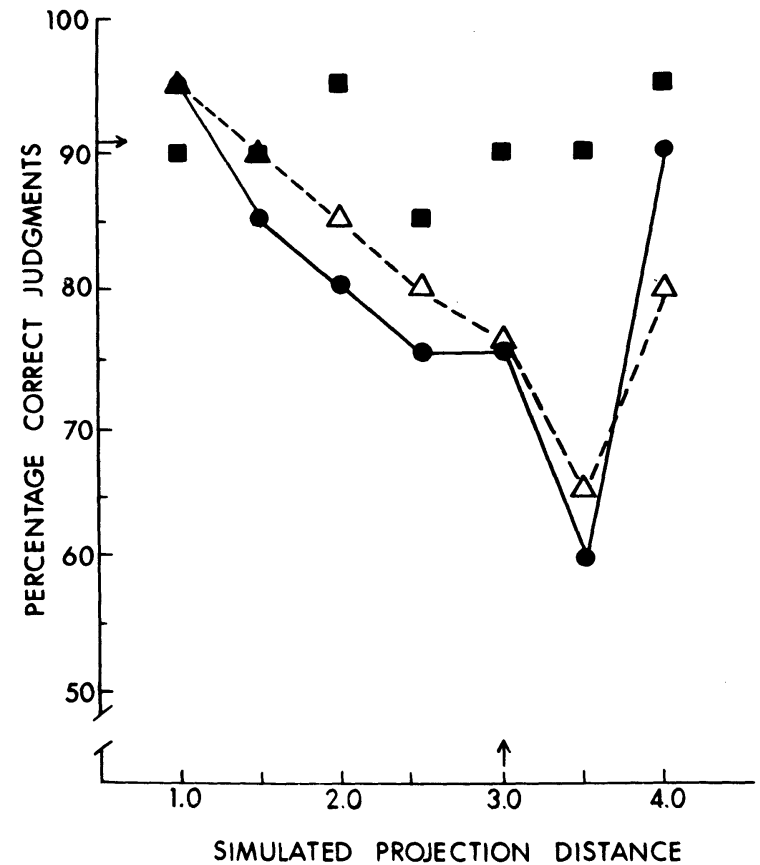

Figure 1. Percentage of correct same-different rotationdirection judgments as a function of simulated projection distance (in sphere radii). Separate curves are for $\mathrm{V}$ spheres (variable spheres) of different sizes: $-s, 2.9 \mathrm{deg} ; \Delta \mathrm{s}, 2 \mathrm{deg}$; -s, 1 deg. Arrow over the abscissa indicates the simulated projection distance of the $\mathrm{C}$ sphere (constant sphere). Arrow beside the ordinate is the mean percent correct for the 2.9-deg V-sphere conditions.

in Figure 1, the percentage of correct same-different judgments for the 2.9-deg V-sphere conditions did not vary much from the mean of $90.7 \%$ (arrow on the ordinate), regardless of simulated projection distance. On the other hand, the data from the intermediate and small spheres reveal a significant effect of simulated projection distance $(p=.034)$. Up to and including a simulated projection distance of 3.5 radii, the percentage of correct judgments declines almost linearly with increasing V-sphere projection distance (decreasing perspective). Beyond 3.5 radii, however, there is an abrupt increase in the percentage of correct same-different judgments. The data for the 1-deg and 2-deg V-sphere size conditions together showed a significant quadratic trend component $(\mathrm{p}<.025)$. It is interesting that the simulated V-sphere projection distance at which the discrimination of relative rotation was lowest was 3.5 radii rather than 3.0 radii, which was the simulated projection distance of the $\mathrm{C}$ sphere (arrow over the abcissa in Figure 1). It should be noted that the functions relating simulated V-sphere projection distance to percent correct same-different judgments for the small and intermediate-sized $\mathrm{V}$ spheres retain the shapes shown in Figure 1 (including the low point at 3.5 sphere radii) even when the data are averaged over both the $10-\mathrm{msec}$ and the 100 -msec IFI conditions.

\section{DISCUSSION}

Three salient discoveries were made in this experiment: 
(1) When as few as 100 moving luminous dots simulated the projections of two transparent spheres rotating in the same locale of the visual field, observers can frequently, although not always, perceptually organize the information into two coherent, simultaneously rotating spheres. (2) The ability of observers to discriminate the relative rotation of the two simulated spheres can be functionally dissociated from the ability to perceive the 3-D structure of the spheres, that is, observers can correctly report the relative rotations of the two spheres even when they cannot subjectively perceive the two spheres themselves. (3) For the conditions reported here, there is no simple monotonic function that relates this relative amounts of perspective in the two spheres to relative rotation discrimination (see Figure 1).

The fact that rotation can be seen in the absence of 3-D structure is similar to the observation that apparent movement, but not spatial structure, is seen during the phi phenomenon. In fact, in the present simulations, the perspective signal is carried by the apparent movement of the dots composing the displays. It is possible that the process yielding the global percept of a 3-D sphere in rotation is based upon the inputs of a short-range apparent movement process (cf. Anstis, 1979; Braddick, 1974; Petersik, Hicks, \& Pantle, 1978).

The last of the three points mentioned above suggests that there is a perceptual interaction of the dynamic perspective information contained in the two simulations such that, when the perspective transformations of the two spheres are similar (i.e., when the simulated projection distances are comparable), the observer has more difficulty segregating the two spheres than when the perspective transformations of the two spheres are different. That the two curves shown in Figure 1 represent true perceptual interactions of the simulated spheres rather than simple discrimination differences based on the varying amount of perspective information contained in the $\mathrm{V}$ sphere as a function of its simulated projection distance is revealed by a comparison of the present results with the single-sphere rotation discrimination data of Petersik (1978). For simulations of individual rotating spheres, Petersik showed that the percentage of correct rotation-direction discriminations declined as a nearly linear function of increasing projection distance over the range of 1.5-12.0 sphere radii. If the percentage of correct samedifferent rotation judgments in the present experiments were based simply upon the discriminability of the rotation of the $\mathrm{V}$ sphere over the various levels of simulated projection distance (as in the case of Petersik, 1978), we would expect to see monotonically declining functions in Figure 1. However, none of the sets of data in Figure 1, including the data for the 2.9-deg $\mathrm{V}$ spheres, shows that pattern of results. Rather, it appears that the relative information in the two spheres interacted to influence the subsequent organization of the relative rotations.

Why did the conditions involving the 2.9-deg V spheres show a different pattern of results than those for the smaller spheres? Pilot studies suggest that the fact that the perspective information in the large $\mathrm{V}$ sphere was distributed over such a large area of the visual field, compared to the area of the $\mathrm{C}$ sphere, actually facilitated the relative organizations of the two spheres. That is, the visual system may have integrated perspective information over different-sized areas of the visual field. Large differences between these spatial areas of integration may yield better percepts. Furthermore, large spheres in general may yield better percepts than small spheres. For example, a single-sphere study by Petersik (1978) showed that 4-deg simulated spheres produced higher percentages of correct rotation-direction judgments than .85-deg spheres.

Finally, the present experiment has again shown the strength of the visual system's tendency to organize 2-D inputs into 3-D percepts (cf. Johansson, 1974, 1977). In most cases, the ability to appropriately group members of a subset of moving dots defined by common dynamic perspective transformations was not hindered by the addition of a second subset of moving dots, also linked by mutual perspective transformations, to the same visual locale.

\section{REFERENCES}

ANstis, S. M. Apparent movement. In R. Held, H. Liebowitz, \& H.-L. Teuber (Eds.), Handbook of sensory physiology (Vol. 8). New York: Springer, 1979.

Braddick, O. A short-range process in apparent movement. Vision Research, 1974, 14, 519-527.

Braunste in, M. L. Depth perception through motion. New York: Academic Press, 1976.

Hershberger, W. A., \& Urban, D. Depth perception from motion parallax in one-dimensional polar projections: Projection versus viewing distance. Journal of Experimental Psychology, 1970, 86, 133-136.

Johansson, G. Projective transformations as determining visual space perception. In R. B. MacLeod \& H. L. Pick (Eds.), Perception: Essays in honor of James J. Gibson. Ithaca, New York: Cornell University Press, 1974.

JoHANSSON, G. Spatial constancy and motion in visual perception. In W. Epstein (Ed.), Stability and constancy in visual perception. New York: Wiley, 1977.

Petersik, J. T. Factors controlling the judgments of rotation direction and depth in certain dynamic perspective transformations. Unpublished doctoral dissertation, Miami University, 1978.

Petersik, J. T. Three-dimensional object constancy: Coherence of a simulated rotating sphere in noise. Perception \& Psychophysics, 1979, 25, 328-335.

Petersik, J. T., Hicks, K. I., \& Pantle, A. J. Apparent movement of successively generated subjective figures. Perception, 1978, 7, 371-383.

\section{NOTE}

1. The term projection refers to the creation of an image on the retina by focusing light reflected from some object or objects in the environment. With a near viewing distance, most of the light reflected from any point on an object reaches the eye at some angle relative to the line of sight. Hence, the point on the 2-D projection plane (in this example, the retina) that is intersected by the light reflected from a particular point on the object is determined by the distance from that point to the projection plane. This means, for any 3-D object, that the projection of a set of "distant" points on an object will differ from the projection of an equivalent set of points located nearer the observer. Projections originating from near viewing distances are known as polar projections. Projections originating from very large viewing distances (i.e., at or beyond optical infinity) are known as parallel projections because the light reflected from objects in the environment is parallel by the time it reaches the eye. Polar projections contain perspective information, whereas parallel projections do not. When an object in the environment moves, one consequence of a polar projection is the well known cue of motion parallax, upon which many of the phenomena related to the present simulations are based. For a more complete discussion of the relationship between polar projections and rotation phenomena, see Braunstein (1976).

(Received for publication May 23, 1979.) 